2018-05-23

\title{
Measurement of Proton Leak in Isolated Mitochondria.
}

\author{
Affourtit, Charles
}

http://hdl.handle.net/10026.1/12335

10.1007/978-1-4939-7831-1_9

Methods in Molecular Biology

Humana Press (Springer Imprint)

All content in PEARL is protected by copyright law. Author manuscripts are made available in accordance with publisher policies. Please cite only the published version using the details provided on the item record or document. In the absence of an open licence (e.g. Creative Commons), permissions for further reuse of content should be sought from the publisher or author. 
In: Carlos M. Palmeira and António J. Moreno (eds.), Mitochondrial Bioenergetics: Methods and Protocols, Methods in Molecular Biology, vol. 1782, 157-170. https://doi.org/10.1007/978-14939-7831-1_9, Springer Science+Business Media, LLC 2018

\title{
Chapter 9
}

\section{Measurement of proton leak in isolated mitochondria}

\author{
Charles Affourtit, Hoi-Shan Wong, and Martin D. Brand
}

\begin{abstract}
Oxidative phosphorylation is an important energy-conserving mechanism coupling mitochondrial electron transfer to ATP synthesis. Coupling between respiration and phosphorylation is not fully efficient due to proton leaks. In this chapter, we present a method to measure proton leak activity in isolated mitochondria. The relative strength of a modular kinetic approach to probe oxidative phosphorylation is emphasized.
\end{abstract}

Key words Mitochondria, Oxygen consumption, Membrane potential, Oxidative phosphorylation, Modular kinetic analysis

Running head: Mitochondrial proton leak

\section{Introduction}

Mitochondria conserve energy as ATP by oxidative phosphorylation. Reducing equivalents derived from the cellular breakdown of carbon-based substrates are donated to the mitochondrial electron transport chain and eventually fully reduce molecular oxygen to water. The energy liberated during this mitochondrial electron transport is used to establish an electrochemical proton gradient across the mitochondrial inner membrane that in turn is used to drive ATP synthesis [1]. The coupling between electron transport and ATP synthesis is not absolute: protons can flow back into the mitochondrial matrix by mechanisms that bypass the ATP synthase [2]. Although proton leak lowers the coupling efficiency of oxidative phosphorylation and thereby decreases the ATP that is generated using carbon fuels, it is not necessarily a wasteful process. Modulation of coupling efficiency by proton leak activity is likely an important mechanism to control cell physiology.

It is clear that mitochondrial proton leak is an important process that, therefore, requires accurate and reliable measurement. In this chapter, we present a protocol to determine proton leak activity in isolated mitochondria. Moreover, we describe a procedure to obtain a system-level, modular kinetic description of oxidative phosphorylation. The methods described may be readily adapted for use with mitochondria from many different sources, but some caution is warranted when applying them to mitochondria from a new source for the first time. The electron leak activity of mitochondria is described elsewhere in this volume under "Plate-based measurement of superoxide/hydrogen peroxide production by isolated mitochondria." 


\section{Materials}

Unless stated otherwise, all chemicals may be purchased from Sigma-Aldrich (St. Louis, $\mathrm{MO})$.

1. Triphenylmethylphosphonium (TPMP)-selective electrode sleeve:

(a) Solutions-Tetraphenylboron $(10 \mathrm{mM})$ in $6 \mathrm{~mL}$ tetrahydrofuran (THF); $1 \mathrm{~g}$ high molecular-weight polyvinylchloride (PVC) in $20 \mathrm{~mL}$ THF; dioctylphthalate (a plasticizer); TPMP (10 $\mathrm{mM}$ in water stored at room temperature).

(b) Equipment-Five glass Petri dishes (100 mm diameter); PVC tubing (4 mm outside diameter); sharp razor blades and scissors.

2. Mitochondrial oxygen consumption and membrane potential:

(a) Oxygen consumption-A water-jacketed Clark oxygen electrode; oxygenpermeable Teflon membrane (see Note 1); an electrode-controller unit and an electronic stirrer, which may all be obtained from Rank Brothers Ltd (Cambridge, UK). A circulating water bath.

(b) Membrane potential $(\Delta \Psi)-\mathrm{A}$ TPMP-selective electrode sleeve; a 2- to 3-cm piece of platinum wire soldered to a screened cable; a solid-state $\mathrm{Ag} / \mathrm{AgCl}$ reference electrode (World Precision Instruments Inc., USA); an adapted oxygen electrode plunger with two additional holes that allow insertion of the $\mathrm{TPMP}^{+}$and $\mathrm{Ag} / \mathrm{AgCl}$ reference electrodes and a $\mathrm{pH}$ meter (we use a $\mathrm{pH}-\mathrm{Amp}$ front-end from AD Instruments, UK).

(c) Data acquisition-A digital recording system comprising a PowerLab analogto-digital signal converter (AD Instruments, UK) linked to any personal computer running LabChart software (AD Instruments, UK).

(d) Assay medium-KHEP $\left(115 \mathrm{mM} \mathrm{KCl}, 10 \mathrm{mM} \mathrm{KH} \mathrm{PO}_{4}, 3 \mathrm{mM}\right.$ HEPES, $1 \mathrm{mM}$ EGTA, $2 \mathrm{mM} \mathrm{MgCl} 2,0.3 \% \mathrm{w} / \mathrm{v}$ bovine serum albumin (BSA), $\mathrm{pH} 7.2$ at 37 ${ }^{\circ} \mathrm{C}$ ) stored at $4{ }^{\circ} \mathrm{C}$ (see Note 2 ).

(e) Calibration-Sodium dithionite (powder) and TPMP ( $1 \mathrm{mM}$ in $\left.\mathrm{H}_{2} \mathrm{O}\right)$, both kept at room temperature, to calibrate the oxygen and TPMP-selective electrodes, respectively.

(f) Respiratory substrates and effectors-Succinate, malate and malonate (stocks at $1 \mathrm{M})$, ADP (100 mM), all prepared in KHEP with $\mathrm{pH}$ adjusted to 7.2 at 37 ${ }^{\circ} \mathrm{C}$. All stocks are stored at $-20^{\circ} \mathrm{C}$. Pyruvate stock at $1 \mathrm{M}$ in KHEP with $\mathrm{pH}$ adjusted to 7.2 at $37^{\circ} \mathrm{C}$ is best made up fresh each day.

(g) Effectors $-1 \mathrm{mg} / \mathrm{mL}$ nigericin, $4 \mathrm{mM}$ rotenone, $1 \mathrm{mg} / \mathrm{mL}$ oligomycin and 1 $\mathrm{mM}$ carbonylcyanide p-trifluoromethoxyphenylhydrazone (FCCP), all prepared in ethanol $(96 \% \mathrm{v} / \mathrm{v})$. All stocks are stored at $-20{ }^{\circ} \mathrm{C}$.

(h) Assays-Automatic pipettes to add KHEP and mitochondria and microsyringes $(5-25 \mu \mathrm{L})$ to add respiratory substrates and effectors.

\section{Methods}

In this chapter, we provide a method to measure proton leak during oxidative phosphorylation in isolated mitochondria. The standard operating procedure in our 
laboratory is to determine the overall rate of proton leak at a range of values of $\Delta \Psi$ to yield the kinetic response of proton leak rate to its driving force, $\Delta \Psi$. Such kinetics are an integral part of a more complete, system-level kinetic description of oxidative phosphorylation that is described below.

1. Mitochondrial isolation from rat skeletal muscle is described elsewhere in this volume under "Measurement of mitochondrial respiration using the XF24 extracellular flux analyzer."

2. Preparation of TPMP-selective electrode sleeves:

(a) Dissolve $1 \mathrm{~g}$ PVC in $20 \mathrm{~mL}$ THF: stir in a covered $50 \mathrm{~mL}$ conical flask until fully dissolved, which takes approximately $1 \mathrm{~h}$.

(b) Combine $6 \mathrm{~mL}$ of $10 \mathrm{mM}$ tetraphenylboron and $20 \mathrm{~mL}$ PVC (both in THF) in a $100-\mathrm{mL}$ flask; mix vigorously.

(c) Add $3 \mathrm{~mL}$ dioctylphthalate and continue mixing.

(d) Divide this mixture equally into five Petri dishes (100 $\mathrm{mm}$ diameter) and allow it to dry on an absolutely level surface in a switched-off fume hood (see Note 3).

(e) Complete evaporation of the solvent, which will take 24 to $48 \mathrm{~h}$, yields a colorless and fairly robust membrane that needs to be attached to an electrode sleeve that should be prepared from a piece of PVC tubing with a $4 \mathrm{~mm}$ outside diameter and approximately $4 \mathrm{~cm}$ length; we use domestic earth (ground) sleeve from an electrical supplier for this purpose (see Note 4).

(f) Put a drop of THF on the membrane and place the PVC sleeve squarely on the drop (alternatively, dip one end of the PVC sleeve in THF and then place on the membrane); support the sleeve until the THF has evaporated and the sleeve is stuck firmly to the membrane. Multiple sleeves can be stuck in each Petri dish; they should be left for 24-48 h to cure.

(g) Cut around the sleeve with a very sharp razor blade and remove it, together with an attached membrane patch, from the Petri dish; trim away any excess membrane with sharp scissors.

(h) Fill the electrode sleeves with TPMP (10 mM) and check for any leaks; these sleeves should be immersed in TPMP $(10 \mathrm{mM})$ for at least $48 \mathrm{~h}$ before use and may be stored dry or in this way for many years.

3. Mitochondrial oxygen consumption:

(a) Oxygen electrode assembly-Set up the oxygen electrode following the manufacturer's instructions (see Note 5). To record dissolved oxygen tensions continuously, connect the electrode controller unit (which applies a polarizing voltage across the oxygen electrode's platinum cathode and $\mathrm{Ag} / \mathrm{AgCl}$ anode and converts oxygen-induced currents into voltages) to the PowerLab analogto-digital signal converter that, in turn, should be linked to a personal computer running LabChart software.

(b) Oxygen electrode calibration-When the set-up's platinum electrode is polarized at $-0.6 \mathrm{~V}$ with respect to the silver electrode, the oxygen-induced current is linearly dependent on the dissolved oxygen tension and a two-point calibration is sufficient to convert the electrical signal into a biologically 
meaningful unit. To calibrate, add $3.5 \mathrm{~mL}$ air-saturated and pre-warmed KHEP to a temperature-controlled $\left(37^{\circ} \mathrm{C}\right)$ and well-stirred oxygen electrode chamber, apply the plunger, and record a voltage that reflects the maximum dissolved oxygen tension; this tension has a value of $406 \mathrm{nmol}$ atomic oxygen per $\mathrm{mL}$ at $37^{\circ} \mathrm{C}$ [3]. To obtain a zero-oxygen signal, remove the plunger and add a few sodium dithionite crystals, which will remove any dissolved oxygen chemically (see Note 6). Between experimental traces, rinse the oxygen electrode vessel thoroughly with distilled water and ethanol (see Note 7).

(c) Calculation of oxygen consumption rates-When isolated mitochondria are incubated with a suitable electron donor, they will exhibit a respiratory activity that is directly proportional to the slope of the time-resolved oxygen trace. LabChart software provides a straightforward option to calculate and visualize the temporal derivative of the oxygen progress curves. The software thus facilitates rapid and objective determination of oxygen consumption rates and, importantly, allows accurate judgements as to whether steady respiratory states have been attained. Mitochondrial respiratory rates should be calculated and presented as specific activities normalized to the amount of mitochondrial protein in the assay to allow direct comparison with published activities.

(d) Coupling of oxidative phosphorylation-It is generally important to ascertain if and to what extent isolated mitochondria have retained coupling between oxygen consumption and ATP synthesis; mitochondrial samples that have lost this coupling are considered poor physiological models and should not be used for proton leak measurement. Oxygen uptake measurements during the various mitochondrial states defined by Chance and Williams [4, 5] provide insight into the degree of coupling, although a more complete understanding can be obtained from modular kinetic experiments that also take $\Delta \Psi$ values into account.

(e) Data collection-Incubate mitochondria $(0.35 \mathrm{mg} / \mathrm{mL}$ if using rat skeletal muscle mitochondria) at $37{ }^{\circ} \mathrm{C}$ in $3.5 \mathrm{~mL}$ KHEP until a stable oxygen electrode signal is achieved (state 1$)$. Then add, sequentially, rotenone ( $4 \mu \mathrm{M})$, succinate $(5 \mathrm{mM})$, and ADP $(50-100 \mu \mathrm{M})$ to, respectively, inhibit respiratory complex I, and to provide an electron donor and a phosphorylation substrate. Well-coupled mitochondria will exhibit a substantial oxygen consumption rate under these conditions (state 3), which will decrease significantly after a short while when all ADP is depleted (state 4). To provoke further state $3 /$ state 4 transitions more ADP aliquots $(50-100 \mu \mathrm{M})$ may be added. The experiment comes to an obvious end when all oxygen has been exhausted and an anaerobic state has been reached (state 5). A respiratory control ratio (RCR) can be derived from the recorded traces, which is a parameter defined as the quotient of oxygen uptake rates under state 3 and state 4 conditions. Good coupling of oxidative phosphorylation will be reflected by a high RCR and we typically observe a value of 4 with rat skeletal muscle mitochondria oxidizing succinate. Alternatively, state 3 respiratory activity can be inhibited by the ATP synthase inhibitor oligomycin $(0.7 \mu \mathrm{g} / \mathrm{mL})$ and then be re-stimulated by the protonophore FCCP $(1.5 \mu \mathrm{M})$. The ratio of FCCP-stimulated and oligomycin-inhibited oxygen uptake rates reflects the extent to which 
mitochondrial electron transfer is coupled to proton translocation. The FCCPoligomycin rate ratio resembles an RCR as it indicates the intactness of the mitochondrial inner membrane, but it differs in that this ratio is not controlled to any extent by the ATP synthase.

The above experiments may also be performed with a combination of pyruvate and malate to engage respiratory complex I in oxidative phosphorylation. These substrates should both be added at $5 \mathrm{mM}$ and rotenone should be omitted from the assay medium.

4. Mitochondrial membrane potential measurements:

(a) TPMP electrode assembly (Fig. 1)-Using an automatic pipette or a microsyringe, add TPMP $(10 \mathrm{mM})$ to a TPMP-sleeve, filling it to about $1 \mathrm{~cm}$ from the top; avoid trapping any air that would prevent proper electrical contact. Attach the filled sleeve to a screened cable such that the connected platinum wire sticks into the TPMP solution, but the soldered connection remains dry. A convenient way to achieve a tight fit is to use a yellow pipette tip, with the very end cut off, as a linker; puncture this pipette tip to prevent pressure changes that may damage the membrane during assembly. Insert both the TPMP-sleeve and the solid-state reference electrode into the oxygen electrode chamber through an adapted plunger and connect them to a voltmeter (a $\mathrm{pH}$ meter is ideal) that is linked to the same PowerLab digital data acquisition system that is used for the oxygen uptake measurements.

(b) TPMP electrode conditioning-Add $3.5 \mathrm{~mL}$ pre-warmed KHEP to a temperature-controlled $\left(37^{\circ} \mathrm{C}\right)$ and well-stirred oxygen electrode chamber, insert the TPMP and reference electrodes, and wait until the signal is stable. Using the $1 \mathrm{mM}$ stock solution, add TPMP to a concentration of $1 \mu \mathrm{M}$, which should cause a substantial electrode response; increasing the TPMP level in 1 $\mu \mathrm{M}$ increments should result in successively smaller responses. The signal should become stable and virtually noise-free at $5 \mu \mathrm{M}$ TPMP. It may take several of these conditioning events, and even an overnight incubation in 2-3 $\mu \mathrm{M}$ TPMP, until a sleeve exhibits drift- and noise-free behaviour and responds to the TPMP dose in a truly logarithmic fashion. Moreover, electrode behaviour tends to improve with use. If such improvement is not observed rapidly, the easiest solution is to try another sleeve. Further troubleshooting suggestions are given in [6]. Following conditioning and between experiments, TPMP sleeves may be stored in medium containing 2-3 $\mu \mathrm{M}$ TPMP for many months.

(c) TPMP electrode calibration and correction for small baseline drift-Although the TPMP electrode exhibits fairly reproducible behavior within daily sets of experimental traces, we nevertheless recommend calibrating its response and assessing possible drift for each trace individually. For a typical experiment, incubate mitochondria $(0.35 \mathrm{mg} / \mathrm{mL})$ at $37{ }^{\circ} \mathrm{C}$ in $3.5 \mathrm{~mL}$ KHEP. Add rotenone $(4 \mu \mathrm{M})$ and nigericin $(350 \mathrm{ng} / \mathrm{mL})$ to inhibit respiratory complex I and collapse the $\mathrm{pH}$ gradient across the mitochondrial inner membrane, respectively. Insert the TPMP and reference electrodes and wait until a stable signal is observed. Using the $1 \mathrm{mM}$ solution, add TPMP in $0.5 \mu \mathrm{M}$ increments 
to a concentration of $2.5 \mu \mathrm{M}$; await a stable signal before each successive addition (see Note 8). Energize the mitochondrial inner membrane by adding succinate $(4 \mathrm{mM})$. If the mitochondria are well coupled, energization will result in a significant TPMP accumulation into the mitochondrial matrix and, therefore, a substantial decrease of the electrode signal. Effectors specific to a particular experiment can then be added; in general, wait until a steady signal is obtained before making additions. The experiment is completed by adding FCCP $(1.5 \mu \mathrm{M})$, which will dissipate the mitochondrial proton motive force (i.e. $\Delta \Psi$ when nigericin is present), will thus cause TPMP release from the mitochondria, and will bring the external TPMP concentration back to $2.5 \mu \mathrm{M}$ within a minute or so. Comparison of the final electrode signal with that observed before succinate addition allows determination of a possible electrode drift rate. If deemed acceptably small, then this rate should be taken into account in the calibration of external TPMP levels. If electrode drift is substantial and/or persistent, its cause(s) should be identified and rectified.

(d) Calculation of membrane potentials-Lipophilic cations such as $\mathrm{TPMP}^{+}$ equilibrate across the mitochondrial inner membrane according to the Nernst equation, $\Delta \Psi=61.5 \times \log \left(\left[\mathrm{TPMP}^{+}\right]_{\text {in }} /\left[\mathrm{TPMP}^{+}\right]_{\text {out }}\right)$ at $37^{\circ} \mathrm{C}$. The membrane potential can thus be calculated from the external (i.e. extra-mitochondrial) and matrix TPMP concentrations. To enable this calculation, measure the signal deflections from the $2.5 \mu \mathrm{M}$ TPMP baseline observed at the various applied TPMP levels and plot this deflection as a function of log [TPMP]. This should yield a linear calibration relation, which can be used to calculate external TPMP concentrations throughout the experiment. Subtracting the external concentration from the $2.5 \mu \mathrm{M}$ TPMP applied provides the TPMP level taken up by mitochondria, which is proportional to its concentration in the matrix. To calculate a reliable concentration, however, it is necessary to know how much of the hydrophobic TPMP is actually freely present within the matrix and how much has in fact bound to mitochondrial membranes. Excellent methods to assess probe binding to membranes and other mitochondrial and/or cellular components have been described [6]. For rat skeletal muscle mitochondria, TPMP-binding has been determined as a function of mitochondrial matrix volume and the obtained correction factor is $0.35 \mathrm{mg}$ mitochondrial protein per $\mu \mathrm{L}$ matrix volume [7]. Multiplication of this correction factor by the TPMP taken up by mitochondria, normalized to the mitochondrial protein present in the assay $(\mathrm{mg} / \mathrm{mL})$, yields the free matrix TPMP concentration. $\Delta \Psi$ values in rat skeletal muscle mitochondria, incubated at $37{ }^{\circ} \mathrm{C}$ and $0.00035 \mathrm{mg} / \mu \mathrm{L}$, can, therefore, be calculated from applied and external TPMP concentrations as:

$$
61.5 \times \log \left(\frac{\left(\left[T P M P^{+}\right]_{\text {applied }}-\left[T P M P^{+}\right]_{\text {external }}\right) \times 0.35}{0.00035 \times\left[T P M P^{+}\right]_{\text {external }}}\right)
$$

5. Proton leak: A modular kinetic description of oxidative phosphorylation.

Oxidative phosphorylation is a process that can be divided conceptually into events that either generate or dissipate the mitochondrial membrane potential [8]. Such a modular view of mitochondrial energy transduction (Fig. 2a) emphasizes 
that steady-state respiratory activities and $\Delta \Psi$ levels result from the kinetic interplay between activity of the mitochondrial electron transfer chain $(\Delta \Psi-$ producer) and the combined activities of the ADP phosphorylation machinery and the proton leak across the mitochondrial inner membrane ( $\Delta \Psi$-consumers). The kinetic dependency of $\Delta \Psi$-establishing and $\Delta \Psi$-dissipating modules on $\Delta \Psi$ is modelled in Fig. $2 \mathrm{~b}$ based on the assumption that the two $\Delta \Psi$-dissipating modules respond hyperbolically (this is not strictly correct, but serves to illustrate the method here). In mitochondria incubated under phosphorylating conditions, a respiratory steady state is reached when the $\Delta \Psi$-establishing rate is equal to the sum of the $\Delta \Psi$-dissipation rates (Fig. 2b, state 3). Steady states achieved upon either inhibition of phosphorylation with oligomycin or dissipation of the mitochondrial proton motive force with excess FCCP are labelled "state 4" and "state F", respectively (Fig. 2b). Note that the oligomycin-inhibited state is generally comparable to the steady state reached in the absence of ADP (state 4). Below, we describe methods to determine modular kinetic relations experimentally.

(a) Theoretical principle-The kinetic behavior of a " $\Delta \Psi$-producer" can be established by specific modulation of a " $\Delta \Psi$-consumer" (Fig. 2c) and, reciprocally, the behavior of a consumer is revealed upon modulation of a producer (Fig. 2d; [8]). In other words, if mitochondrial respiration is titrated under state 3 conditions with sub-saturating amounts of an electron transfer chain inhibitor, the kinetics with respect to $\Delta \Psi$ of the total $\Delta \Psi$-dissipating activity (i.e. leak + phosphorylation) are revealed. If this titration is performed in the presence of oligomycin, the kinetics of proton leak alone are obtained, which may be subtracted from the overall $\Delta \Psi$-dissipating kinetics to reveal the behavior of the ADP-phosphorylation module. If respiration is titrated with FCCP, either in the presence or the absence of oligomycin, the kinetic dependency of the respiratory chain on $\Delta \Psi$ is established. During these respiration titrations, successive steady states will be attained in which both the oxygen uptake rate (flux) and $\Delta \Psi$ (intermediate concentration) should be measured simultaneously. Plotting oxygen consumption rates as a function of the concomitant $\Delta \Psi$ values allows empirical construction of modular kinetic plots.

(b) Proton leak kinetics-Add mitochondria $(0.35 \mathrm{mg} / \mathrm{mL})$ to $3.5 \mathrm{~mL} \mathrm{KHEP} \mathrm{in} \mathrm{an}$ oxygen electrode vessel incubated at $37{ }^{\circ} \mathrm{C}$ in the presence of rotenone $(4 \mu \mathrm{M})$, nigericin $(350 \mathrm{ng} / \mathrm{mL})$, and oligomycin $(0.7 \mu \mathrm{g} / \mathrm{mL})$. Apply the plunger, insert the TPMP and reference electrodes, and record both oxygen and TPMP signals. Make five sequential TPMP additions of $0.5 \mu \mathrm{M}$ each. Add succinate (4 $\mathrm{mM}$ ) to initiate oligomycin-resistant respiration and to energize the mitochondria. When the oxygen uptake rate and TPMP signal have stabilized, make at least five successive malonate additions increasing its concentration gradually to approximately $0.5 \mathrm{mM}$; finish the trace by adding FCCP $(1.5 \mu \mathrm{M})$. Calculate specific oxygen consumption rates (see Note 9) and plot as a function of the concomitant $\Delta \Psi$ values. The leak rate dependency on $\Delta \Psi$ may be modelled approximately by an exponential expression. 
(c) Phosphorylation kinetics-Perform an experiment identical to that described for the proton leak kinetics, but omit oligomycin from the incubation mixture. Instead, include sufficient ADP or an ADP-regenerating system to enable phosphorylating respiration throughout the trace. The data obtained in this experiment represent the kinetic dependency of the sum of phosphorylation and leak activities on $\Delta \Psi$. Subtraction of proton leak kinetics yields the $\Delta \Psi$ dependency of the phosphorylation module alone, which may also be approximated by an exponential expression.

(d) Electron transfer chain kinetics-Add mitochondria $(0.35 \mathrm{mg} / \mathrm{mL})$ to $3.5 \mathrm{~mL}$ KHEP in an oxygen electrode vessel incubated at $37{ }^{\circ} \mathrm{C}$ in the presence of rotenone $(4 \mu \mathrm{M})$, nigericin $(350 \mathrm{ng} / \mathrm{mL})$, and oligomycin $(0.7 \mu \mathrm{g} / \mathrm{mL})$. Add TPMP to $2.5 \mu \mathrm{M}$ in five equimolar increments and add succinate $(4 \mathrm{mM})$ to initiate respiration. When the oxygen uptake rate and TPMP signal have stabilized, make at least 5 successive FCCP additions, increasing its concentration gradually to approximately $0.8 \mu \mathrm{M}$; finish the trace by adding surplus FCCP $(1.5 \mu \mathrm{M})$. Calculate specific oxygen consumption rates and plot as a function of the concomitant $\Delta \Psi$ values. The electron transfer chain rate dependency on $\Delta \Psi$ may be modelled approximately by a hyperbolic expression.

Simultaneous measurement of oxygen consumption and $\Delta \Psi$ provides considerably more insight into oxidative phosphorylation than individual determination of these parameters [1]. Mitochondrial coupling efficiency is better understood from modular kinetic information than from respiration alone since the kinetic behavior of the respective modules explains the exact nature of a particular RCR and, moreover, identifies the underlying cause(s) of possible differences in RCRs between experimental conditions and systems [2]. Mitochondrial proton leak activity can be compared in a meaningful manner between experimental systems and conditions, because the oligomycin-insensitive respiratory rates can be corrected for possible differences in the leak's driving force, $\Delta \Psi$. Similarly, activity of the other kinetic modules can be probed at identical $\Delta \Psi$ values [9]. Site(s) at which suspected effectors of oxidative phosphorylation act can be identified unambiguously [10]. Conclusive evidence can be obtained as to the role played by poorly characterized proteins in oxidative phosphorylation [11]. Elasticities of all kinetics modules to $\Delta \Psi$ can be calculated, which allows control and regulation of oxidative phosphorylation to be quantified [8].

\section{Notes}

1. The standard oxygen-permeable Teflon membrane may be substituted with high-sensitivity membrane (YSI Life Sciences, Ohio, USA) to decrease the response time of the oxygen electrode.

2. Bovine serum albumin should be added to the KHEP assay medium on the day of use; sprinkle the required amount $(0.3 \%, \mathrm{w} / \mathrm{v})$ on top of the solution and let it dissolve without stirring to prevent clotting. 
3. Modern fume hoods may sometimes not be switched off; to prevent hasty evaporation and surface rippling of the membrane, cover the Petri dishes in this case with lids but leave small openings to enable drying.

4. When cutting PVC tubing, it is pivotal to get a very clean and straight cut as the sleeve will not stick to the membrane otherwise. The likelihood of obtaining such cuts is maximized when performed by two persons: one pulls the tubing as tightly as possible and the other cuts it very quickly and cleanly with a sharp razor blade.

5. Whether or not the assembled setup detects oxygen and responds rapidly to changes in oxygen concentration can be checked conveniently by switching off the electronic stirrer temporarily, which should cause a substantial and fast signal deflection. Lack of stirring means that oxygen level near the platinum cathode and the bulk assay medium are no longer kept in rapid equilibrium and, therefore, that the oxygen uptake at the cathode will lead quickly to localized oxygen deprivation.

6. Sodium dithionite cannot always be used to deplete oxygen from the assay medium as it may interfere with other measurements such as the detection of TPMP. An alternative way to obtain the zero-oxygen signal is to rely on mitochondrial respiration to consume all oxygen and use the anaerobic signal achieved at the end of an experimental trace. If this "biological zero" is known from previous experiments to be similar to the signal obtained from an unpolarized electrode, this "electrical zero" could be used as a quick-and-dirty approximation of a zero-oxygen signal.

7. Some respiratory effectors (e.g. rotenone and FCCP) are hard to wash away between experimental traces as they tend to stick to the plastic oxygen electrode vessel. Even when a glass vessel is used to reduce this sticking issue, it may sometimes be wise to rinse the electrode chamber between traces with a concentrated $(3 \%, \mathrm{w} / \mathrm{v})$ bovine serum albumin solution or with a suspension of frozen-thawed mitochondria that were left over from earlier experiments. Bovine serum albumin and mitochondria will bind many possible nasties and using them as a mop will greatly reduce the likelihood of effector carry-over.

8. Once in a while, the TPMP electrode will exhibit small drift. It is important, however, to distinguish such drift from a lack of biological steady state as it is possible that $\Delta \Psi$ is changing slowly because of alteration in electron transfer chain, proton leak and/or phosphorylation activities. Changes in these processes, however, will also likely be reflected in altered oxygen consumption rates. An important indication, therefore, that a slowly changing TPMP signal reflects biological reality and is not merely due to electrode drift, is a concomitantly changing oxygen electrode signal.

9. Oligomycin-insensitive oxygen uptake activities are converted to proton flux rates by multiplying them by appropriate $\mathrm{H}^{+} / \mathrm{O}$ ratios, which are 6 and 10 during the oxidation of succinate and pyruvate/malate, respectively. ADPphosphorylation rates are obtained by multiplying the leak-corrected state 3 
respiratory rates by the appropriate $\mathrm{P} / \mathrm{O}$ ratios, which are 1.64 during oxidation of succinate and 2.73 during oxidation of pyruvate plus malate [12].

\section{Acknowledgements}

C.A. thanks Julie Buckingham for useful advice on the preparation of TPMP sleeves.

\section{References}

1. Nicholls DG, Ferguson SJ (2013) Bioenergetics, 4th edn. Academic Press, Elsevier, Amsterdam

2. Brand MD (2005) The efficiency and plasticity of mitochondrial energy transduction. Biochem Soc Trans 33:897904. doi: 10.1042/BST20050897

3. Reynafarje B, Costa LE, Lehninger AL (1985) $\mathrm{O}_{2}$ solubility in aqueous media determined by a kinetic method. Anal Biochem 145:406-418.

4. Chance B, Williams GR (1955) Respiratory enzymes in oxidative phosphorylation. III. The steady state. J Biol Chem 217:409-427.

5. Brand MD, Nicholls DG (2011) Assessing mitochondrial dysfunction in cells. Biochem J 435:297-312. doi: 10.1042/BJ20110162

6. Brand MD (1995) Measurement of mitochondrial protonmotive force. In: Brown GC, Cooper CE (eds) Bioenergetics: a practical approach. IRL, Oxford, pp 39-62

7. Rolfe DFS, Hulbert AJ, Brand MD (1994) Characteristics of mitochondrial proton leak and control of oxidative phosphorylation in the major oxygenconsuming tissues of the rat. Biochim Biophys Acta BBA - Bioenerg 1188:405416. doi: 10.1016/0005-2728(94)90062-0
8. Brand MD (1998) Top-down elasticity analysis and its application to energy metabolism in isolated mitochondria and intact cells. Mol Cell Biochem 184:13-20.

9. Brand MD, Affourtit C, Esteves TC, Green K, Lambert AJ, Miwa S, Pakay JL, Parker N (2004) Mitochondrial superoxide: production, biological effects, and activation of uncoupling proteins. Free Radic Biol Med 37:755-767. doi: 10.1016/j.freeradbiomed.2004.05.034

10. Affourtit C, Brand MD (2008) Uncoupling protein-2 contributes significantly to high mitochondrial proton leak in INS-1E insulinoma cells and attenuates glucose-stimulated insulin secretion. Biochem J 409:199-204. doi: 10.1042/BJ20070954

11. Dröge W (2002) Free radicals in the physiological control of cell function. Physiol Rev 82:47-95. doi: 10.1152/physrev.00018.2001

12. Mookerjee SA, Gerencser AA, Nicholls DG, Brand MD (2017) Quantifying intracellular rates of glycolytic and oxidative ATP production and consumption using extracellular flux measurements. J Biol Chem 292:71897207. doi: 10.1074/jbc.M116.774471 


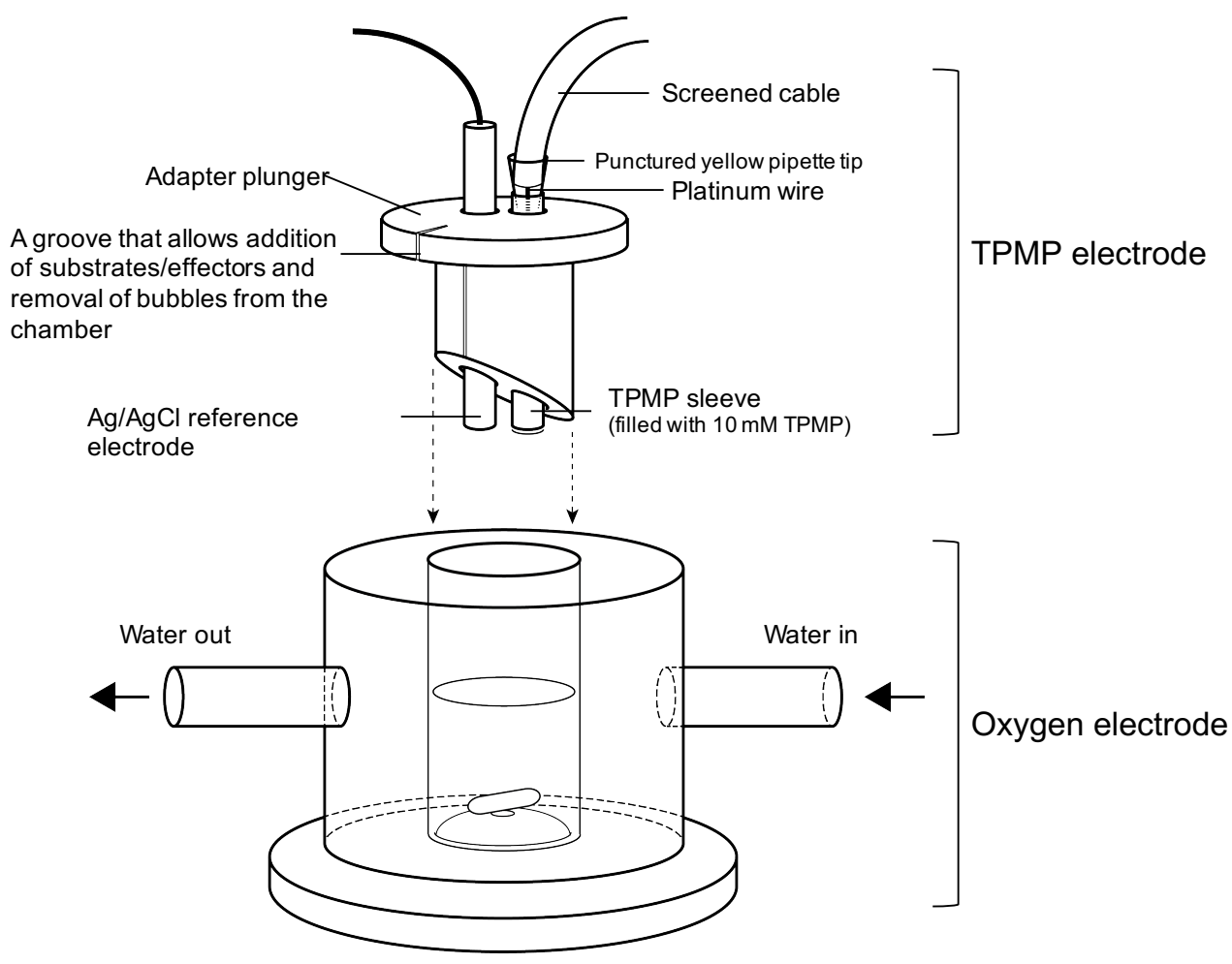

Fig. 1 TPMP electrode and oxygen electrode assembly. The TPMP electrode and oxygen electrode are set up as described in Subheading 3 

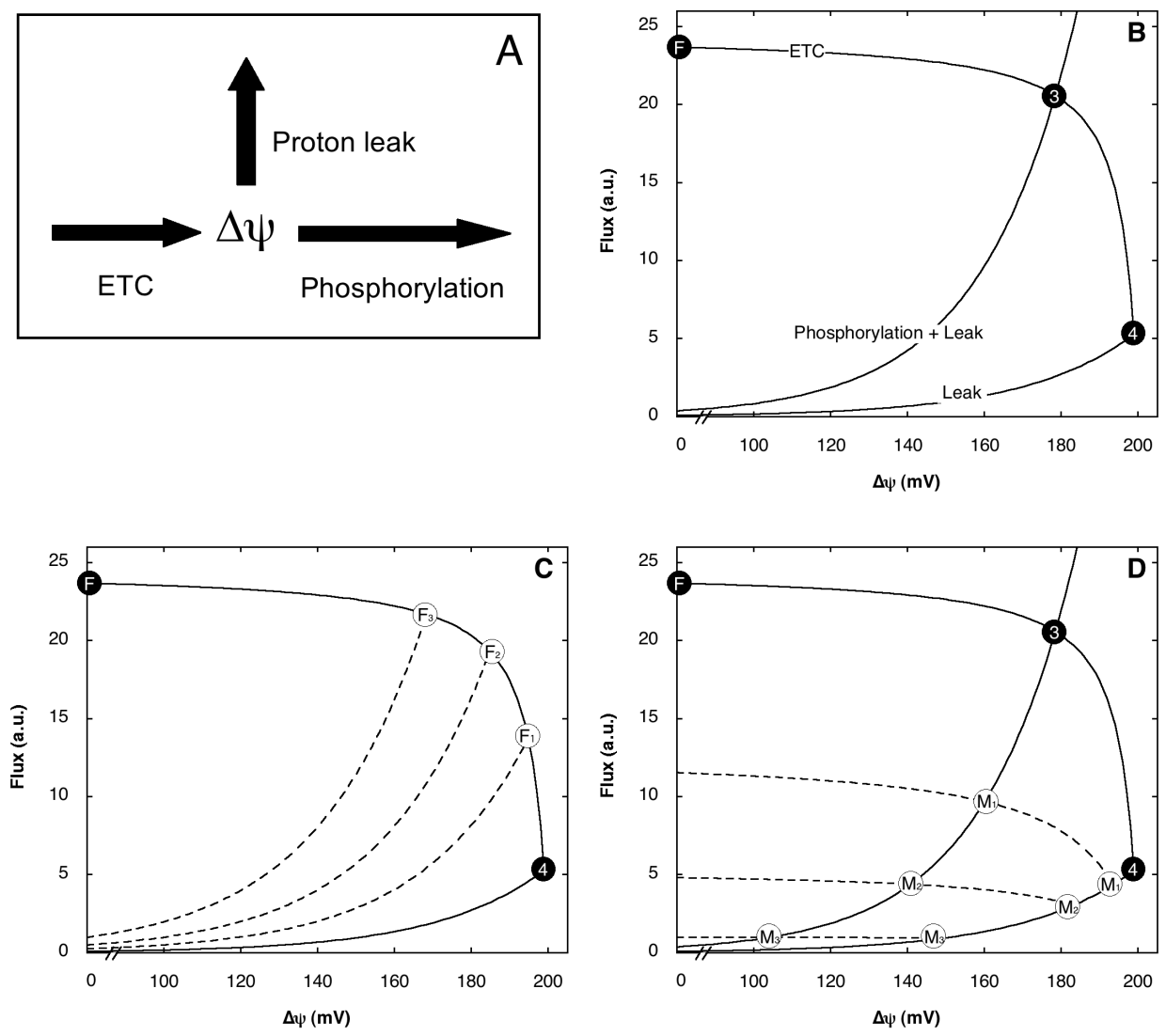

Fig. 2 Modelled modular kinetics during oxidative phosphorylation in isolated mitochondria. (a) Oxidative phosphorylation considered from a top-down perspective as an interaction between processes that establish $\Delta \Psi$ (electron transport chain, ETC) and those that dissipate it (proton leak and phosphorylation). (b) The kinetic dependency of both the ETC and the sum of proton leak and phosphorylation rates (fluxes expressed in arbitrary units) on $\Delta \Psi$ was modelled using exponential (proton leak, phosphorylation) and hyperbolic (ETC) equations. The model assumes zero $\Delta \mathrm{pH}$ (presence of nigericin) so that the proton motive force equals $\Delta \Psi$. Steady states are achieved when $\Delta \Psi$-establishing flux equals total $\Delta \Psi$-dissipating flux, which is reflected by intersections of the curves describing the behaviour of the respective rates. Modelled steady states include those reached in the presence (state 3 ) and the absence of ADP (state 4) as well as that achieved in the presence of FCCP (state F). (c) Titration of oligomycin-insensitive respiration rate with sub-saturating amounts of FCCP increases proton leak activity specifically. New steady states will thus be achieved that reflect the altered proton leak kinetics and the unaffected ETC kinetics (states F1-F3). (d) Titration of succinate oxidation with subsaturating amounts of malonate inhibits ETC activity specifically. In this case, new steady states will be reached that reflect the altered ETC kinetics and the unaffected $\Delta \Psi$ dissipating kinetics (states M1-M3). Malonate titrations under phosphorylating conditions thus reveal the kinetic behavior of the sum of $\Delta \Psi$-dissipating behavior and titrations in the presence of oligomycin reveal the kinetic behavior of proton leak alone 